\title{
Initial test results on bolometers for the Planck high frequency instrument
}

\author{
Warren A. Holmes, ${ }^{1, *}$ James J. Bock, ${ }^{1,2}$ Brendan P. Crill, ${ }^{1,3}$ Timothy C. Koch, ${ }^{1}$ \\ William C. Jones, ${ }^{1,4}$ Andrew E. Lange,,$^{1,2}$ and Christopher G. Paine ${ }^{1}$ \\ 'Jet Propulsion Laboratory, California Institute of Technology, 4800 Oak Grove Dr., Pasadena, California 91109, USA \\ ${ }^{2}$ California Institute of Technology, Mail Stop 59-33, Pasadena, California 91125, USA \\ ${ }^{3}$ Infrared Processing and Analysis Center, California Institute of Technology, Pasadena, California 91125, USA \\ ${ }^{4}$ Department of Physics, Princeton University, Princeton, New Jersey 08544, USA \\ ${ }^{*}$ Corresponding author: Warren.A.Holmes@ jpl.nasa.gov
}

Received 6 June 2008; accepted 11 August 2008;

posted 10 October 2008 (Doc. ID 97085); published 4 November 2008

\begin{abstract}
We summarize the fabrication, flight qualification, and dark performance of bolometers completed at the Jet Propulsion Laboratory for the High Frequency Instrument (HFI) of the joint ESA/NASA Herschel/ Planck mission to be launched in 2009. The HFI is a multicolor focal plane which consists of 52 bolometers operated at $100 \mathrm{mK}$. Each bolometer is mounted to a feedhorn-filter assembly which defines one of six frequency bands centered between 100-857 GHz. Four detectors in each of five bands from $143-857 \mathrm{GHz}$ are coupled to both linear polarizations and thus measure the total intensity. In addition, eight detectors in each of four bands $(100,143,217$, and $353 \mathrm{GHz})$ couple only to a single linear polarization and thus provide measurements of the Stokes parameters, Q and U, as well as the total intensity. The measured noise equivalent power (NEP) of all detectors is at or below the background limit for the telescope and time constants are a few $\mathrm{ms}$, short enough to resolve point sources as the 5 to 9 arc min beams move across the sky at $1 \mathrm{rpm}$. (C) 2008 Optical Society of America
\end{abstract}

OCIS codes: $\quad 040.0040,040.1240,040.2235$.

\section{Introduction}

The ESA/NASA Planck Surveyor mission, to be launched in 2009, will perform a full sky survey of the $\mathrm{mm}$-wave universe [1]. The Planck spacecraft consists of a telescope radiatively cooled to $\sim 35 \mathrm{~K}$ and a cryocooler chain that cools a high electron mobility transistor (HEMT) array receiver to $20 \mathrm{~K}$ and the High Frequency Instrument (HFI) to $100 \mathrm{mK}$. Each detector is a micromachined spiderweb bolometer (SWB) $[2,3]$ or pair of polarization sensitive bolometers (PSBs) [4,5] mounted in a compact module complete with filters to attenuate RF power. The SWB are mounted to feedhorns with bandpass filters that define five bands, centered at $143,217,353,545$,

0003-6935/08/325996-13\$15.00/0

(C) 2008 Optical Society of America and $857 \mathrm{GHz}$. The PSBs, similarly, are mounted to feedhorns with bandpass filters that define four bands centered at 100,143, 217, and $353 \mathrm{GHz}$. The SWB and PSBs in each band have a detector noise equivalent power (NEP) so that the in flight sensitivity is limited by the photon noise, in Fig. 1, which is the quadrature sum of contributions from the background produced by the sky, the cold telescope, and the cryogenic filter stages in the instrument. Each bolometer, feedhorn, and filter assembly, cooled to $100 \mathrm{mK}$, is mounted at the focal plane of a telescope with 9,7 , and 5 arc min resolution for the bands 100 , 143 , and $\geq 217 \mathrm{GHz}$, respectively. The spacecraft samples the sky by spinning at $1 \mathrm{rpm}$ at its orbit at the Earth-Sun L2 Lagrange point. The response time $\tau$ of all bolometers, summarized in Fig. 2 , is fast enough to resolve point sources at the resolution of the telescope. Here we report on the completion of the build, 


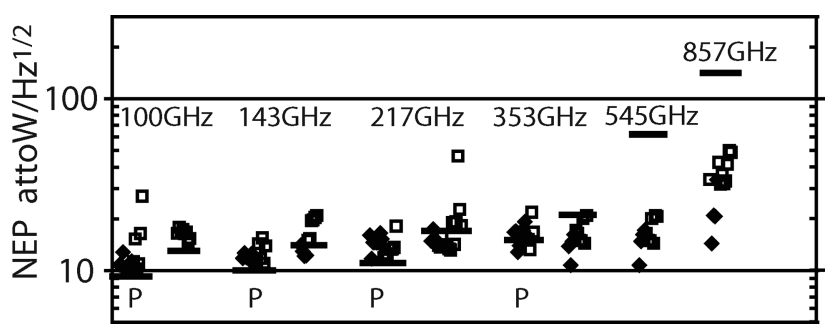

Fig. 1. Measured detector dark NEP including $6.5 \mathrm{nV} / \sqrt{\mathrm{Hz}}$ amplifier noise at nominal bias for each frequency band. Solid symbols are the NEP for detectors installed in the focal plane. The open symbols are the NEP spare bolometers. The solid lines indicate the photon background limit from a $35 \mathrm{~K}$ telescope and astrophysical sources in each band for a 30\% bandwidth and 30\% in band optical efficiency. Unpolarized detectors at $100 \mathrm{GHz}$ were made and delivered but were replaced by polarized detectors.

flight qualification, and cryogenic testing of the detector modules for HFI.

There were two production and test cycles at the Jet Propulsion Laboratory (JPL). First, cryogenic qualification modules (CQM) were made as pathfinders for the microfabrication, module build, and testing [6]. Second, a much larger quantity of Planck flight modules (PFM) were made and tested. Each bolometer module was subjected to acceptance level random vibration, shock, and cryogenic thermal cycling tests to ensure survival through integration, test, and launch in the spacecraft. After environmental testing, the noise and response time of the detectors, without the cold optics, was measured at $100 \mathrm{mK}$. Most modules were then shipped to University of Cardiff, Wales (UCW) for intergration with the feedhorn and bandpass assemblies and optical testing. A small set of modules that passed environmental and functional tests were selected for qualification and destructive tests. These tests included elevated random vibration levels and extended thermal cycling or accelerated aging to simulate $\sim 5$ years of shelf life. After nondestructive qualification testing, the bolometers were retested to verify that the functional performance was unchanged. In this paper, we describe the results of these tests performed at JPL. The optical performance measured at UCW, when integrated with the feedhorns and filters, and

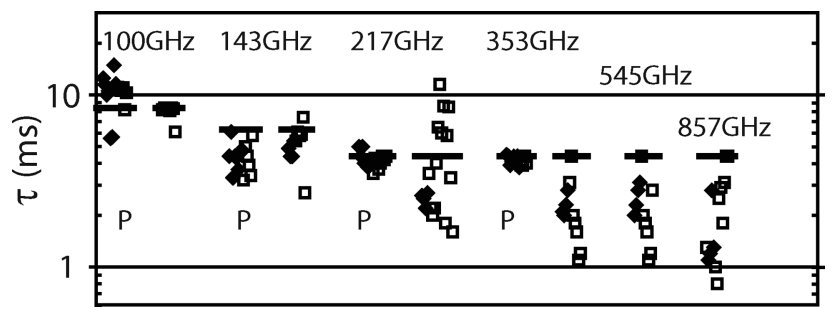

Fig. 2. Measured detector response time $\tau$ at nominal bias for each frequency band. Solid symbols are the $\tau$ for detectors installed in the focal plane. The open symbols are the $\tau$ of the spare bolometers. The solid lines indicate a response time equal to time for the beam center to travel the beam width divided by $\pi$ at a spacecraft rotation rate of $1 \mathrm{rpm}$. Unpolarized detectors at $100 \mathrm{GHz}$ were made and delivered but were replaced by polarized detectors. system performance measured at Institut d'Astrophysique Spatiale (IAS, Orsay, France) when integrated with the flight electronics and $100 \mathrm{mK}$ cooler, will be described elsewhere.

\section{Bolometer Theory}

Here we review the basic formula necessary to discuss the design and testing of the Planck bolometers [7-10]. This theory was established for DC biased bolometers and proved to be applicable in our case. A bolometer is a broadband thermal detector. The lumped element thermal model shown in Fig. 3 is a useful description of the bolometer behavior. A metallized absorber mesh with heat capacity $C(T)$ is weakly linked by thermal conductance $G(T)$ to a heat sink at temperature $T_{s}$. The metal mesh absorbs millimeter wave radiation, which increases the absorber temperature $T$. The absorber temperature $T$ is measured as the change in resistance $R(T)$ of semiconducting neutron transmutation doped (NTD) Ge thermometer [11] biased at constant current $I_{b}$. The thermometer voltage is measured using cooled, low noise junction field effect transistor preamplifiers. The voltage responsivity,

$$
S=\frac{\alpha \sqrt{P_{b} R(T)}}{G(T)+i 2 \pi f C-\alpha P_{b}+2 \alpha P_{b} R /\left(R+R_{l}\right)},
$$

in $\mathrm{V} / \mathrm{W}$, is the ratio of a small voltage change to a small signal power oscillating at frequency $f$, where $\alpha=1 / R \times d R / d T, P_{b}=R(T) I_{b}^{2}$ is the electrical bias power, and $R_{l}$ is the value of the load resistor $R_{l} \gg$ $R(T)$ used in the bias circuit shown in Fig. $\underline{4}$. The response time is

$$
\tau=\frac{C(T)}{G(T)-\alpha P_{b}+2 \alpha P_{b} R /\left(R+R_{l}\right)} .
$$

The detector NEP, in $\mathrm{W} / \sqrt{\mathrm{Hz}}$, is defined as the optical signal which is equal to the noise in a $1 \mathrm{~Hz}$ amplifier bandwidth at the output. The square of the total noise is the sum of the square of the uncorrelated sources

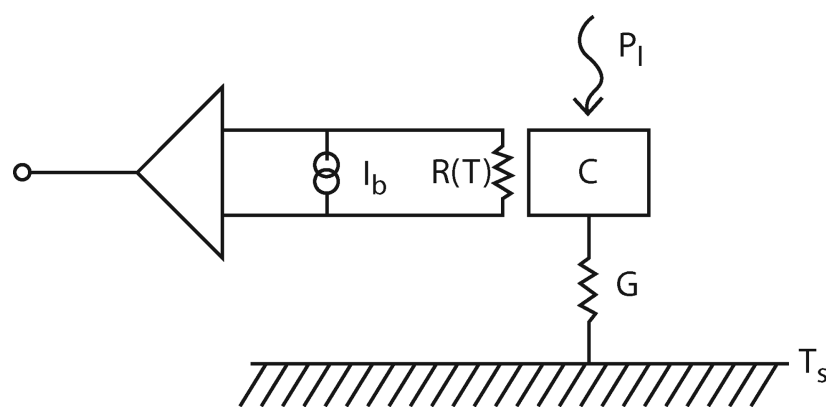

Fig. 3. Schematic model of a single lumped element bolometer. Optical power $P_{l}$ is absorbed in heat capacity $C$, which is linked to heat sink at temperature $T_{s}$ by a thermal link with thermal conductance $G$. The temperature of the absorber is measured using a resistive thermometer $R(T)$ biased with constant current $I_{b}$ and readout with a low noise voltage amplifier. 


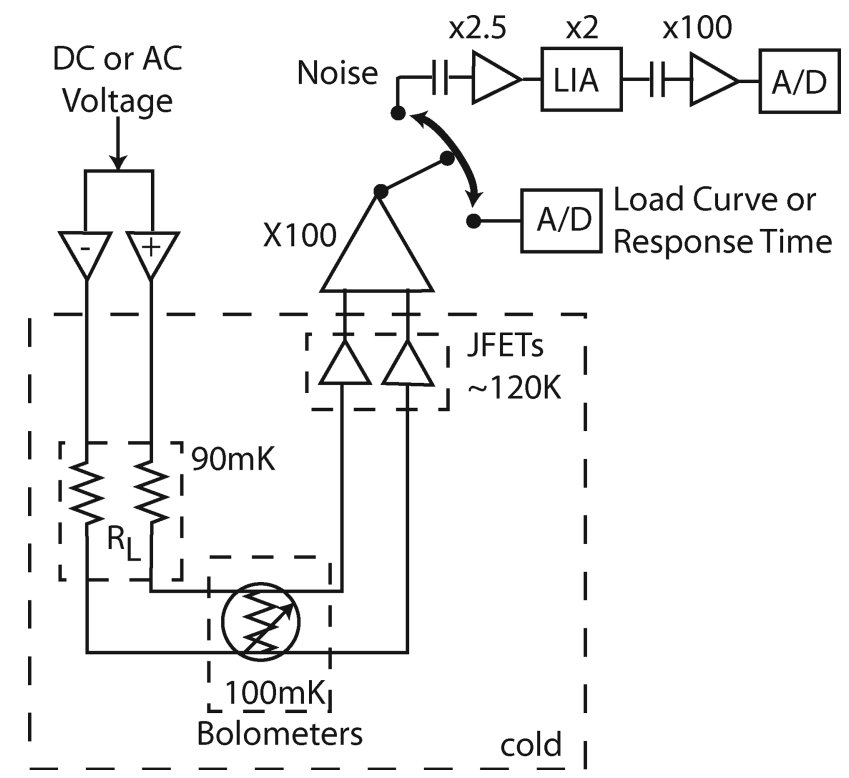

Fig. 4. Amplifier chain used for measurement of bolometer performance at $100 \mathrm{mK}$. A DC input voltage and a DC coupling is used for load curve and response time measurements. An AC input voltage, AC coupling, and additional gain stages are used for the noise measurements.

$$
\begin{aligned}
\epsilon^{2} \mathrm{NEP}_{\text {tot }}^{2}= & \gamma_{1} 4 k T^{2} G(T)+\gamma_{2} \frac{4 k T R(T)}{S^{2}} \\
& +\frac{e_{n}^{2}+\gamma_{3} R^{2}(T) i_{n}^{2}}{S^{2}}+\mathrm{NEP}_{\text {photon }}^{2}+\frac{v_{\text {excess }}^{2}}{S^{2}}
\end{aligned}
$$

where $\epsilon$ is the optical efficiency of the bolometer. The factors $\gamma_{i}$ represent nonequillibrium effects [9]. The first term, $\gamma_{1} 4 k T^{2} G(T)$, represents thermal fluctuation noise from phonon exchange between the absorber and the heat sink. The factor $\gamma_{1}$ is important when the thermal gradient along the thermal link is large. The second term, $\gamma_{2} 4 k T R / S^{2}$, is the Johnson noise of the thermometer referred to the bolometer input by dividing by $S^{2}$. The third term, $\left(e_{n}^{2}+\right.$ $\left.\gamma_{3} R^{2}(T) i_{n}^{2}\right) S^{2}$, is the amplifier noise referred to the bolometer input, in $\mathrm{V}^{2} / \mathrm{Hz}$. The fourth and fifth terms represent the incident power photon noise [12], in $\mathrm{W}^{2} / \mathrm{Hz}$, and any excess noise in the bolometer or readout, respectively, in $\mathrm{V}^{2} / \mathrm{Hz}$.

\section{Bolometer Description}

\section{A. Millimeter Wave Absorber}

The mm-wave absorber for SWBs and PSBs is a free standing circular, metallized $\mathrm{Si}-\mathrm{N}$ micromesh suspended by $\mathrm{Si}-\mathrm{N}$ beams [3,5]. The micromesh absorber grid element width is $\sim 5-10 \mu \mathrm{m}$ wide and $<1 \mu \mathrm{m}$ thick. The grid spacing, $>50 \mu \mathrm{m}$, is chosen so that it absorbs mm-waves with high efficiency but has a much smaller physical area which reduces, significantly, the cross section to cosmic ray particles and shorter wavelength photons.
For the SWBs, which are sensitive to total power from both incident polarizations, the micromesh is a radial, spider web pattern with a diameter of $2 \lambda$, where $\lambda$ is the wavelength at the center of the bandpass. The sheet impedance of the metallization is optimized for peak absorption in a corrugated feedhorn fed cavity formed by the backface of the feedhorn and a $1 / 4$ wave backshort behind the absorber.

For the PSBs, which are sensitive to a single linear polarization, the micromesh is in a square grid pattern and metallized along only one direction and the thermistor is mounted at the edge of the absorber. Two PSBs are mounted in the same feedhorn cavity in a manner to detect each orthogonal linear polarization. The grid spacing and sheet impedance of the metallization are optimized not only for peak absorption but also to minimize response to the orthogonal polarization (cross polar response) [5]. For both the SWBs and PSBs, measures are taken to ensure that the absorber and thermistor are isothermal.

\section{B. Thermal Behavior}

The sensitivity of bolometers is fundamentally limited by statistical fluctuations of the radiation power coming from observed source and thermal emission from the cryogenic stages of the instrument. This noise limit is commonly called the BLIP noise. The representative background power $P_{l}$ and noise $\mathrm{NEP}_{\mathrm{BLIP}}$ were computed for each band [1] and used to design the bolometers. Detailed discussions of bolometer optimization are given in several references $[3,8,10,13]$. Here we summarize these optimization routines as they were used for Planck HFI. The value of $G$ for each band is bound between a maximum value set by the detector noise $\mathrm{NEP}_{\mathrm{BLIP}} \sim \sqrt{G_{\text {max }}}$ and a minimum value $G_{\min }$ set either by the response time specification $\tau \sim C / G_{\min }$ or to avoid saturation at the background power loading. The design target $G$ for the bolometers in each band was set as the geometric mean of $G_{\max }$ and $G_{\min }$. The thermal conductance through the $\mathrm{Si}-\mathrm{N}$ support beams was negligible compared to the target $G$ for each band. This allowed very good matching of the sensitivity of devices within each band.

\section{Temperature Sensor}

The bolometer temperature is measured using a neutron transmutation doped (NTD) Ge semiconducting thermistor. Neutron transmutation doping produces a very uniform and reproducible p-type doping density [11]. The resistance as a function of temperature is described by phonon assisted variable range hopping $(\mathrm{VRH})$ law $R(T)=(l / A) \rho_{o} \exp \left(\sqrt{\left(T_{g} / T\right)}\right)$. The values of $\rho_{o}$ and $T_{g}$ are specific to a given doping density. The material used for Planck has a doping density $n=5.6 \times 10^{16} \mathrm{~cm}^{-3}$, which has $T_{g} \sim 18 \mathrm{~K}$ and $\rho_{o} \sim 10^{-1} \mathrm{Ohm} \mathrm{cm}$. The $l / A=0.1 \mu \mathrm{m}^{-1}$ ratio is chosen so that the resistance $\sim 10 \mathrm{M} \Omega$ at the operating temperature and bias is matched to the JFET preamplifiers with a voltage noise of $e_{n}<6.5 \mathrm{nV} / \sqrt{\mathrm{Hz}}$. At the chosen $l / A$ ratio, the NTD Ge volume, 
$30 \mu \mathrm{m} \times 100 \mu \mathrm{m} \times 300 \mu \mathrm{m}$, was large enough so that the chips could be manually placed on the bolometers, yet also small enough that the heat capacity was acceptably low. At the chosen thickness, $30 \mu \mathrm{m}$, excess noise due to conduction by phonon assisted $\mathrm{VRH}$, as measured in much thinner $(0.1 \mu \mathrm{m}$ thick $)$ ion implanted Si thermistors [14], is negligible for the NTD Ge used for Planck. Low noise ohmic contact is made to the NTD Ge through boron implanted and metallized contact pads [11] and indium bump bonded onto contact pads on the absorber.

\section{Bolometer Module Assembly}

The Si-N micromesh bolometer die were fabricated at JPL Microdevices Laboratory (MDL) [15]. The bolometers were produced in two batches of several wafers. The processing starts with commercial "SOI" silicon wafers [16], 6 in. in diameter, which have a $\sim 1 \mu \mathrm{m}$ thick $\overline{\mathrm{SiO}}_{2}$ layer buried beneath a $\sim 1 \mu \mathrm{m}$ thick, Si top layer on one side of the wafer. On each wafer, a Si-N film, $\sim 1 \mu \mathrm{m}$ thick, was grown on both sides of the wafer. Gold with a titanium adhesion layer was deposited and patterned on the side of the wafer with the buried oxide in three steps to define the mm-wave absorber, the thermal link to the absorber, and the thermalization bars and contact pads for wire bonding and the indium bump bonds. For the first batch, the Cryogenic Qualification Model (CQM) bolometers, the indium bump bonds were deposited directly on the gold contact layers. For the second batch, the Planck Flight Model (PFM) bolometers, a nickel layer was deposited and patterned on the gold contacts prior to deposition of indium bump bonds [15]. Once the metallization was complete, the $\mathrm{Si}-\mathrm{N}$ film was patterned by plasma etching to define the micromesh absorber pattern and the etch windows on the backside of the wafer. Each wafer was then diced into segments referred to as slices. The final steps, indium bump bonding and "the release" process, were completed one slice at a time. Special tooling produced a controlled force to press the indium bumps uniformly to the contact pads on the NTD Ge chip, as shown in Fig. 5. Then,

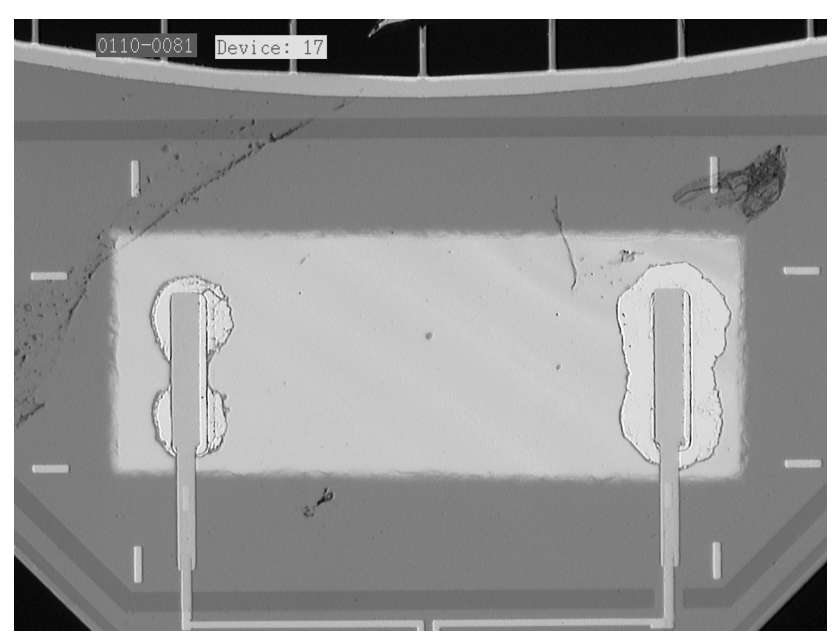

Fig. 5. NTD Ge bump bonded to the bolometer. This photograph was taken from the backside of the bolometer to show the compression of indium bumps.

the slice was etched from the backside to remove the silicon to define the free standing metalized $\mathrm{Si}-\mathrm{N}$ absorber mesh with NTD Ge thermistor. The slice release was staggered, as shown in Table 1 , to allow timely evaluation of the yield to the release process and to performance requirements. The quality of CQM wafers (PL217353A, PL217353B, 1905, and $1909)$ and PFM wafers $(1917,1921,1923,1876,1878$, 1884 and 0081) was comparable so both were used for the flight build. Slice B from 1921 yielded $100 \mathrm{GHz}$ SWBs. Slices C and D from 1921 and 1923 yielded $143 \mathrm{GHz}$ SWBs. Slices TR, R, and $\mathrm{T}$ from PL217353A and PL217353B and slices E and F from wafers 1917, 1921, and 1923 yielded 217 and 353 GHZ SWBs. Slices TR and T from wafers 1905 and 1909 yielded 545 and $857 \mathrm{GHz}$ SWBs. Slices A and $\mathrm{B}$ from wafer 1876 and 1884 yielded $100 \mathrm{GHz}$ PSBs. Slices C and D from wafers 1876, 1884, and 0081 yielded 143, 217, and $353 \mathrm{GHz}$ PSBs. All slices on wafer 1878 and slices A and B from wafer 0081 had so much excess heat capacity that the response times of these devices were $2-5 \times$ too slow for Planck.

Table 1. Wafers Processed for Planck Flight Bolometers ${ }^{a}$

\begin{tabular}{|c|c|c|c|c|c|c|}
\hline \multirow[b]{2}{*}{ Wafer } & \multicolumn{6}{|c|}{ Slice } \\
\hline & $\mathrm{A}(\mathrm{TR})$ & $\mathrm{B}(\mathrm{TL})$ & $\mathrm{C}(\mathrm{BR})$ & $\mathrm{D}(\mathrm{BL})$ & $\mathrm{E}(\mathrm{T})$ & $\mathrm{F}(\mathrm{R})$ \\
\hline PL217353A & $12 / 12 / 01$ & $\mathrm{x}$ & $\mathrm{x}$ & $\mathrm{x}$ & $10 / 24 / 01$ & $\mathrm{x}$ \\
\hline PL217353B & $\mathrm{x}$ & $\mathrm{x}$ & $\mathrm{x}$ & $\mathrm{x}$ & $8 / 21 / 01$ & $8 / 21 / 01$ \\
\hline 1905 & 2/13/03 & $\mathrm{x}$ & $\mathrm{x}$ & $\mathrm{x}$ & - & - \\
\hline 1909 & $2 / 28 / 03$ & $\mathrm{x}$ & $3 / 1 / 02$ & $\mathrm{x}$ & - & - \\
\hline 1917 & $\mathrm{x}$ & $\mathrm{x}$ & $\mathrm{x}$ & $\mathrm{x}$ & $10 / 17 / 02$ & $10 / 17 / 02$ \\
\hline 1921 & $\mathrm{x}$ & $8 / 8 / 02$ & $8 / 23 / 02$ & 8/23/02 & $\mathrm{x}$ & $9 / 13 / 03$ \\
\hline 1923 & $\mathrm{x}$ & $\mathrm{x}$ & $5 / 6 / 03$ & $\mathrm{x}$ & $3 / 3 / 02$ & $3 / 24 / 03$ \\
\hline 1876 & $3 / 7 / 03$ & $2 / 9 / 04$ & 6/16/03 & 7/15/03 & - & - \\
\hline 1878 & $10 / 29 / 02$ & $1 / 31 / 03$ & $2 / 28 / 03$ & $\mathrm{x}$ & - & - \\
\hline 1884 & $2 / 3 / 04$ & $6 / 4 / 04$ & $11 / 20 / 03$ & $\mathrm{x}$ & - & - \\
\hline 0081 & $4 / 3 / 03$ & $4 / 3 / 03$ & $4 / 16 / 03$ & $5 / 12 / 03$ & - & - \\
\hline
\end{tabular}

${ }^{a}$ CQM wafer slice names are shown in the parentheses. PFM slices were named A-F except for wafers 1876, 1878, 1884, and 0081, which have slices A-D only. An ' $\mathrm{x}$ ' in the table denotes a process failure at the slice level. 
Released bolometer die were mounted into bolometer modules at the JPL hybrid electronics facility. The module parts were machined from beryllium copper and electroplated with gold. The module for the SWB consists of a backshort, a printed wiring board (PWB) with an RF filter circuit and connection terminal to the bolometer and a cover [17]. The bolometer die is located over the $1 / 4$ wave relief machined into the "backshort" part of the module and glued into place with epoxy. The bond area for every bolometer was measured by taking an image at an infrared wavelength where the silicon is transparent. Bond areas range from $0.5-8 \mathrm{~mm}^{2}$. Wired bonded gold wires connect contact pads on the bolometer die to filter capacitors glued to the PWB mounted in the bolometer module on the opposite side of the backshort from the bolometer. The PWB board contains surface mount inductors and a ground plane that, with the capacitors and bolometer, form a filter to attenuate RF before it is rectified at the bolometer. The cover is installed onto the backshort to protect the PWB and support a connector. A high quality picture, Fig. $\underline{6}$, is taken of each final assembly.

The bolometer module for the PSB consists of a housing, a backshort and a cover. One PSB is epoxied into the housing and a second into the backshort as shown in Fig. 7. The alignment of each PSB is fixed by a notch in the bolometer die and a raised "key" feature in the housing and backshort. Reference pictures of the mounted bolometers are taken before mating. The polarization sensitivity of the backshort, or aft, bolometer is orthogonal to that of the housing, or fore, bolometer. The angle of the housing relative to the backshort is fixed using dowel pins. The parallelism is controlled by precise machining and careful mating of the housing and backshort. When assembled, the aft and fore bolometer absorbers are $60-100 \mu \mathrm{m}$ apart. Unlike the SWB, the last corrugation of the feedhorn optics is machined into the PSB housing to allow more precise control and verification of the bolometer positions inside the cavity. The PSB module contains a similar PWB and RF attenuator circuit as the SWB module. The completed PSB module is baked to relieve residual strain in the cured epoxy joint. The angle of each PSB relative to one another and to the fiducial mark is measured to a

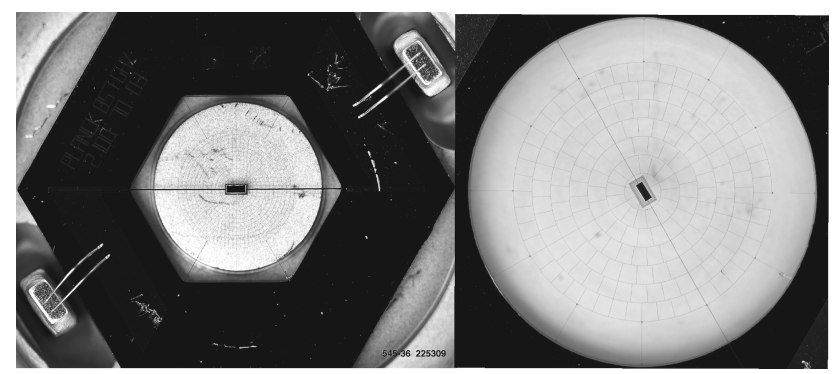

Fig. 6. Completed multimode $545 \mathrm{GHz}$ (left) and single mode $100 \mathrm{GHz}$ (right) SWB bolometer modules just prior to shipping. The raised $1 / 4$ wave backshort is visible behind the absorber mesh of the $545 \mathrm{GHz}$ bolometer.

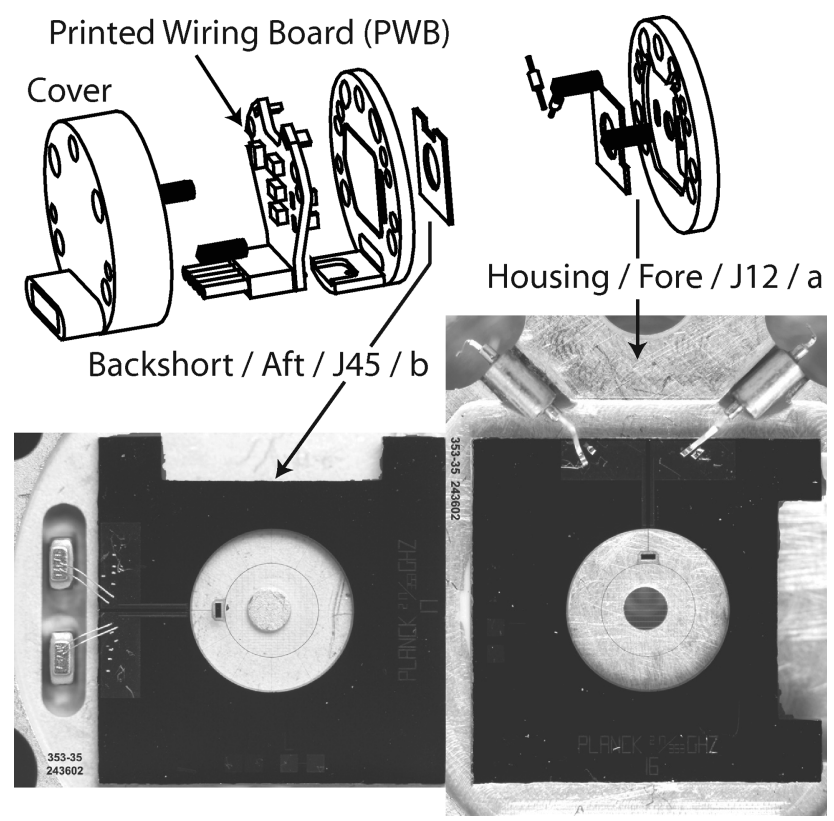

Fig. 7. PSB bolometer pair epoxied in the module parts prior to mating. At the top is an exploded view of the assembly. Alignment pins, shown in solid black, fix the aft and fore bolometer assemblies to an angular precision of $<0.1^{\circ}$. On the right, the feedhorn aperture can be seen through the fore bolometer in the housing. On the left, the $1 / 4$ wave backshort can be seen through the aft bolometer absorber mesh. The NTD Ge chip is bump bonded at the edge of each absorber far away from the aperture and backshort outside of the region with large polarized electric field strength.

precision and accuracy of $<0.1^{\circ}$. The stability of this angle measurement was confirmed on a subset of samples throughout the environmental and cryogenic testing. Attempts were made to match bolometers in PSB by selecting die from neighboring locations on the same wafer. A large database referenced to the bolometer die wafer and serial number was built and maintained to properly link wafer fabrication data, module assembly history, and test data.

\section{Module Environmental Testing}

\section{A. Thermal Cycle}

All assembled bolometer modules were cycled several times between 300 and $90 \mathrm{~K}$ prior to delivery. Qualification modules were cycled an additional 80 times between $300 \mathrm{~K}$ or $345 \mathrm{~K}$ and $<90 \mathrm{~K}$ to prove the design was robust. For each thermal cycle test, up to 16 SWB or 8 PSB bolometer modules were mounted on a thermally isolated stage in an evacuated liquid nitrogen cryostat. The bolometers were cooled at $\sim 2 \mathrm{~K} / \mathrm{min}$ using a liquid nitrogen flow through a heat exchanger on the stage. Once the bolometer temperature was $<90 \mathrm{~K}$, the nitrogen flow continued for $10 \mathrm{~min}$ and was then shut off by closing a solenoid actuated valve. The bolometers were heated to 300 or $345 \mathrm{~K}$ at a rate of $\sim 2 \mathrm{~K} / \mathrm{min}$ using a heater. Once at the high temperature, the heater was turned off. After a 10 min dwell, the liquid nitrogen flow valve was opened to cool the bolometers. 
Bolometer resistance was measured every minute using peak bias current of $0.1 \mu \mathrm{A}$. The entire cycling and measurement process was automated using LabVIEW software and commercial computer interface hardware. More than 3000 joint-cryogenic thermal cycles were completed without a single bolometer module component or assembly failure.

\section{B. Vibration Testing}

All bolometer modules were subjected to random vibration and simulated shock along all three orthogonal axes as expected for launch on an Ariane V rocket. The random vibration acceleration spectral density (ASD) for each test increased from 10 to $75 \mathrm{~Hz}$ at $\sim+12 \mathrm{~dB} /$ octave to a fixed value from 75 to $400 \mathrm{~Hz}$ and then decreased at $\sim-12 \mathrm{~dB}$ /octave from 400 to $2000 \mathrm{~Hz}$. Bolometer modules were tested at several vibration levels as summarized in Table 2 . The equivalent static load used for structural margin calculations is $3 \times$ the root mean square (rms) acceleration computed from the measured acceleration spectral density during test. This static load is applied for a total of $1 \mathrm{~s}$ during each $1 \mathrm{~min}$ of vibration. The shock of $1000 \mathrm{~g}$, where $g=9.8 \mathrm{~m} / \mathrm{s}^{2}$, was applied for $<10 \mathrm{~ms}$. Random vibration and shock levels were applied using shake tables at one of several facilities. At each test facility, the bolometers were protected from contamination and electrostatic discharge (ESD) inside an hermetically sealed aluminum carrier. The bolometer resistance was measured before and after the test in the controlled environment at JPL hybrid facility. Three modules, all of which passed acceptance testing, were tested beyond the qualification acceleration until failure. The results of the destructive tests are described in Table 2.

\section{Epoxy Joint Characterization}

The strength of the epoxy bond was characterized by shear and random vibration tests using residual bolometer die and modules. Two types of epoxy joints were prepared for test. The first type was made using the nominal cure used for all bolometers. The second type was prepared with a different "cold" cure process, which was desirable since it simplified the build process. The bond area of epoxy was measured using an infrared camera that provided a high contrast image of the epoxy relative to the surrounding metal housing. Each joint was die shear tested until failure.

Table 2. Summary of Bolometer Random Vibration Testing ${ }^{a}$

\begin{tabular}{lcccl}
\hline $\begin{array}{l}\text { Qty } \\
\text { Tested }\end{array}$ & $\begin{array}{c}\text { Equivalent } \\
\text { Static Load }\end{array}$ & $\begin{array}{c}\text { Duration } \\
\text { (minutes) }\end{array}$ & Failures & \multicolumn{1}{c}{ Comments } \\
\hline 86 & $30 \mathrm{~g}$ & 3 & 0 & PFM PSB acceptance \\
68 & $36 \mathrm{~g}$ & 3 & 0 & $\begin{array}{l}\text { PFM SWB acceptance } \\
15\end{array}$ \\
$51 \mathrm{~g}$ & 6 & 1 & $\begin{array}{l}\text { Previously tested at } \\
\text { (m) }\end{array}$ \\
4 & $51 \mathrm{~g}$ & 12 & 3 & $\begin{array}{l}\text { Before and after qual } \\
\text { thermal cycle }\end{array}$ \\
1 & $71 \mathrm{~g}$ & 2 & 0 & Broke at $150 \mathrm{~g}$ \\
1 & $150 \mathrm{~g}$ & 1 & 1 & \\
\hline
\end{tabular}

Using the lowest bond measured strength, the structural margin of safety for failure to shear stress is $>20$ for the smallest bond areas $\sim 1 \mathrm{~mm}^{2}$ at our static equivalent qualification acceleration. Typical structural margins are $\sim 100$ and even higher for peel stress. The pattern of the epoxy break, in bolometers vibration tested to failure, shows failure due only to shear stress, as expected. The epoxy joints prepared using the cold cure were on average a factor 100 weaker per unit area but still showed a positive margin of safety. However, the joints cold cured had a variability in strength of nearly $100 \%$ about the average. A few bolometers were mounted in modules using the cold cure process and with large bond areas. The failure rate during qualification vibration testing was still unacceptably large and consistent with the shear test results. So, the cold cure process was not used for flight units.

\section{Accelerated Aging}

Unlike many ground or suborbital applications, the HFI bolometers were made many years prior to launch. Diffusion of the metallized contact pads on the $\mathrm{Si}-\mathrm{N}$ mesh into the indium bump bonds or low level corrosion due to insufficient rinsing during the photolithography were concerns at the beginning of the build. To test the design and lithographic processes for these effects, three completed bolometer modules and two devices consisting of only a suspended NTD Ge thermistor with no absorber were held at high temperature to simulate 5.5 years of shelf life. The resistance of the bolometers was measured hourly during the test using a bias current of $0.1-1 \mu \mathrm{A}$. The resistance of the bolometers and one of the absorberless devices measured before, during, and after the test were unchanged. No faults or changes were found in any lithographic features and indium bump bonds after the test. However, the second of the two absorberless devices measured open circuit after $48 \mathrm{~h}$ in the test chamber. The open circuit was caused by a fracture in a $\mathrm{Si}-\mathrm{N}$ beam with a readout lead, but was not unique to the aging test. Two of the four surviving devices were destructively tested at random vibration levels at and above the qualification level. The remaining two devices have stable optical efficiency and low noise performance at $100 \mathrm{mK}$ as of this publication.

\section{Performance Testing}

The performance of every bolometer was measured using a dilution refrigerator insert equipped with temperature controlled stages, a light pipe with IR blocking filters, and 24 parallel voltage readout channels [18]. The bolometers were mounted at the bottom of the dilution refrigerator insert, shown in Fig. 8. During installation and removal from the dilution insert, filtered and ionized air is blown over the work area. Once all bolometers were mounted, the volume around the bolometers was hermetically sealed with an indium O-ring to prevent excess IR 


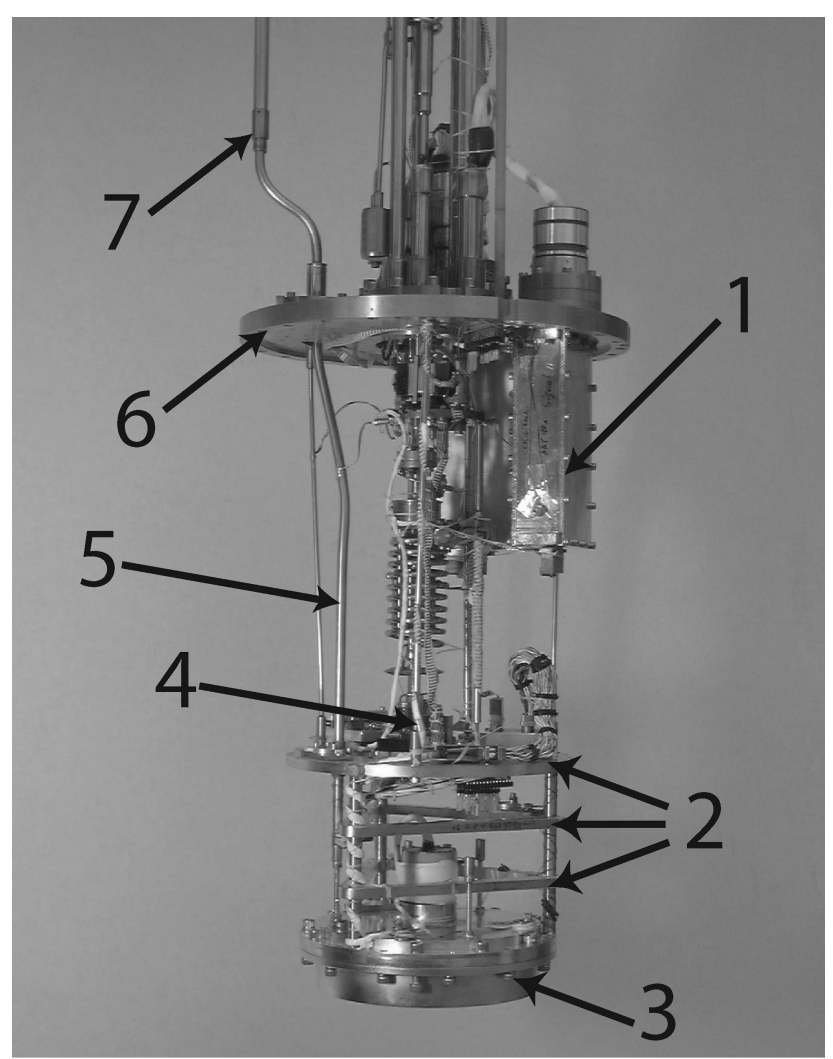

Fig. 8. Photo of the dilution refrigerator insert used for testing at $100 \mathrm{mK}$. The JFET box (1), thermal isolation stages (2), hermetic bolometer can (3), mixing chamber (4), and light pipe (5) are enclosed in a vacuum can sealed using indium wire to the insert flange (6) and immersed in liquid helium. Infrared filters or a brass plug can be changed while the insert is at $4 \mathrm{~K}$, at the threaded light pipe joint (7).

loading and incidental contamination, for example, from vacuum pump oil or adsorbed helium gas.

\section{A. Readout Electronics}

The readout amplifiers for the $100 \mathrm{mK}$ testbed, shown in Fig. 4, were adapted from ground based bolometric array receivers [17,19]. The bolometers were biased by applying voltage to cold load resistors $R_{l}=20 \mathrm{M} \Omega$ mounted on the mixing chamber of the dilution fridge to produce current $I_{b}$. The output voltage of each bolometer was buffered by cold JFET follower with low voltage noise $e_{n}<6.5 \mathrm{nV} / \sqrt{\mathrm{Hz}}$ and current noise $i_{n}<0.6 \mathrm{fA} / \sqrt{\mathrm{Hz}}$. The JFET preamplifier was located near the bolometer stage to minimize the stray capacitance $<150 \mathrm{pF}$ and therefore allows a bandwidth $>200 \mathrm{~Hz}$ at bolometer operating resistance of $\sim 10 \mathrm{M} \Omega$. Additional $\mathrm{RF}$ filters were used for all bolometer bias and readout wires routed from the cold JFET module. A second DC coupled amplification stage with gain of 100 , mounted over the warm feedthrough on the dilution insert top flange, was used to measure the $V_{o}\left(I_{b}\right)$ for each bolometer. For bolometer noise measurements, a square wave at $\sim 41-46 \mathrm{~Hz}$ was applied to the load resistor and bolometer circuit using Stanford Research System (SRS) 360 function generator [20]. The output vol- tage was synchronously demodulated by an AC coupled lock-in amplifier stage. The total voltage gain and bandwidth for the noise measurements are 60,000 and $\sim 8 \mathrm{~Hz}$, respectively. Voltages for all measurements were digitized using a National Instruments data acquisition card [21]. The tests were automated using LabVIEW software.

\section{B. $100 \mathrm{mK}$ Testbed Calibration}

Calibration of the $100 \mathrm{mK}$ testbed readout electronics required calibrated thermometers, measurement of the load resistors, DC and AC gain, and tracking of the time base for response time measurements. All gain calibrations were performed on the integrated data acquisition system, including the digitizer and software control. Each calibration was cross checked against design values and bench top component tests and is traceable to a NIST standard.

Primary thermometers used for the $100 \mathrm{mK}$ testbed were germanium resistance thermometers (GRT) purchased from Lakeshore Cryotronics [22]. Each GRT was heat sunk by mounting it inside a copper bobbin and wrapping $\sim 50 \mathrm{~mm}$ of the $\sim 0.1 \mathrm{~mm}$ diameter current and voltage lead manganin wires around the bobbin. Two primary thermometers were mounted on the bolometer stage. Each of the five primary thermometers was heat sunk aggressively at several locations, including the mixing chamber, still, $1 \mathrm{~K}$ pot and helium cold plate to prevent temperature measurement offset from heat conducted from room temperature down the readout wires. The primary thermometer resistances were measured using an AVS47 resistance bridge [23] with a bias voltage of $100 \mu \mathrm{V}$ and 8 channel multiplexer. Two new thermometers were purchased and installed midway through our 4 year test program. The seven thermometers were rotated among the five locations in the testbed at $\sim 9$ month intervals as an additional validation of our temperature measurements. The calibrations of the new and old thermometers agreed to $\sim 1 \mathrm{mK}$.

More sensitive secondary thermometers and a readout system were used for the thermal control loop. These thermometers were NTD Ge thermistors purchased from Haller Beeman Associates [24]. They were mounted near each Lakeshore GRT primary thermometer on each stage. The secondary thermometers were measured using an AC current bias applied using load resistors mounted to the mixing chamber. The frequency of the thermometer bias was always chosen to be exactly $4 \times$ the frequency for the bolometer bias during noise measurement since the synchronous demodulators naturally reject even multiples of the bias frequency. Each thermometer voltage was measured using a dedicated amplifier consisting of a cold JFET preamplifier and a lock-in stage mounted to the warm flange of the dilution fridge insert. The secondary thermometer channels were calibrated using the primary GRTs. The calibration points were fixed output voltages of the entire readout chain. We increased the current bias 
with increasing temperature to maintain an output voltage in the range $0.1-2 \mathrm{~V}$. Typically, the mixing chamber and one of the thermal intercepts, items (4) and (2), respectively, in Fig. 8, were regulated at fixed temperature using a $\overline{2} \Omega k$ heater and TS530 PID controllers [25]. The bolometer stage, item (3) in Fig. 8 , was uncontrolled and allowed to reach equilibrium with the thermal intercept prior to any measurements of the bolometers. The precision of the temperature control was routinely $<1 \mu \mathrm{K} / \sqrt{\mathrm{Hz}}$.

The load resistor for each channel was measured at room temperature using a four wire technique to a precision of $0.01 \%$ with a commercial calibrated ohmmeter. The gain of each DC coupled amplifier chain, $g_{\mathrm{DC} i}$ at operating temperature, was calibrated using reference resistors $R_{i}$ cooled to $100 \mathrm{mK}$. Each $g_{\mathrm{DC} i}$ is consistent with the product of the designed and measured gains of each amplification stage.

The transfer function of each AC coupled amplifier chain was calibrated by measuring the noise at the output, $v_{\mathrm{ACout}}^{2}$, generated from a reference noise source at the input. The reference noise source was provided by the Johnson noise, $4 k T R_{i}$, from resistors with known $R_{i}$ at temperature $T$. Each time series file was checked for glitches. Files without glitches were Fourier transformed, averaged and fit to the formula

$$
v_{\mathrm{ACout}}^{2}=g_{\mathrm{AC} i}^{2}\left(v_{\mathrm{white}}^{2}+v_{\mathrm{excess}}^{2} / f\right)\left[\frac{\omega^{2} \tau_{\mathrm{AC} i}^{2}}{1+\omega^{2} \tau_{\mathrm{AC} i}^{2}}\right],
$$

where the gain $g_{\mathrm{AC} i} \sim 60,000$, the white noise $v_{\text {white }}^{2}$, the single pole time constant of the AC coupling filter, $\tau_{\mathrm{AC} i}$, and $v_{\text {excess }}$ are fitting parameters. Noise was measured at several temperatures, resistance values and bias levels spanning the range applied to the bolometers. At typical biases and output noise voltages used for bolometer tests, the gain nonlinearities were negligible $\ll 1 \%$. The values of $v_{\text {white }}^{2}$ were then fit to $v_{\text {white }}^{2}=4 k T R_{i}+e_{n}^{2}+i_{n}^{2} R_{i}^{2}$, to determine the amplifier voltage $e_{n} \sim 6.5 \mathrm{nV} / \sqrt{\mathrm{Hz}}$ and current noise $i_{n}<0.6 \mathrm{fA} / \sqrt{\mathrm{Hz}}$. A small contribution to $v_{\text {excess }}$ measured at zero bias was due entirely to the lock-in amplifier.

There was an additional bias dependent noise measured using the fixed resistors with a $1 / f$ knee at $\sim 100 \mathrm{mHz}$. This bias dependent excess noise was present only in tests of the fully integrated system and was not measured in isolated component tests. This $1 / f$ knee at $\sim 100 \mathrm{mHz}$ present in the testbed electronics prevented verification of bolometer noise down to $16 \mathrm{mHz}$ as specified for Planck.

The time base for the measurements, primarily the thermal response time, was provided by monitoring a reference sine wave from the SRS360 function generator [20], at a frequency in the range $41-46 \mathrm{~Hz}$ fixed at a single value during measurements. The accuracy of the frequency is well below the $<10 \%$ mea- surement accuracy implied by the requirements for the bolometer response time.

\section{Static Thermal Properties}

Detailed discussions of the electronic behavior of bolometers are available in the literature $[7,8,10,26]$. As discussed in these reference papers, here we summarize the measurements and analyses we performed to fully characterize the bolometers so that we can use bolometer theory to predict performance under varying conditions as requested by the Planck instrument team. First, we measured bolometer load curves $V_{o}\left(I_{b}\right)$ using the electronics configured with a DC bias current. We stepped through a series of voltages applied to the load resistor and bolometer, $V_{b}$, at both positive and negative values of $V_{b}$ centered about $V_{b}=0$. The time between each new bias point was $>1 \mathrm{~s}$ to ensure that the bolometer time response did not affect the measurements. Then, we fit the slope at low bias through $V_{b}=0$, to determine $R$ at each measurement temperature $T$. The $R(T)$ computed from the slope near $V_{b}=0$ was fit to the form $R(T)=R_{\text {leads }}+R_{o} \exp \left(\sqrt{T_{g} / T}\right)$, where $R_{\text {leads }}$ is the lead resistance and $R_{o}$ and $T_{g}$ are standard fitting parameters for variable range hopping (VRH) conduction. These fits were good over the entire temperature range indicating that stray $R F$ power or infrared power was negligible. The signal to noise was typically $\sim 1 \%$. However, intermittent electronic offsets in readout of the primary thermometers, but not the secondary thermometers, resulted in absolute uncertainties of a few $\mathrm{mK}$. To estimate the absolute accuracy of the thermometry, we define a reference resistance at $100 \mathrm{mK}$ of $R_{c}=52.35 \Omega M$ from the unweighted average $R_{o c}=73.3 \Omega$ and $T_{g c}=$ $18.16 \mathrm{~K}$ obtained from the fits of $R(T)$ measured for 123 different bolometers. Next, we use the measured resistance of each NTD when the primary thermometers indicate the temperature is $100 \mathrm{mK}$. Then, we compute the NTD temperature using the reference NTD parameters $R_{o c}$ and $T_{g c}$ for each bolometer, and take the difference between the primary thermometer temperature and the NTD temperature. A Gaussian fit to these binned differences has a width $\pm 6 \mathrm{mK}$ which bounds the temperature measurement error to $\pm 6 \%$ at $100 \mathrm{mK}$. This accuracy will be improved by follow up measurements at UCW and at the integrated instrument test at IAS, Orsay, France.

We subtracted the offset fit from the low bias range and computed the bolometer voltage $V_{o}$ and the actual current bias using the formula $I_{b}=\left(V_{b}-V_{o}\right) /$ $R_{l}$. Using the VRH fit parameters and value of $R\left(I_{b}\right)=V_{o}\left(I_{b}\right) / I_{b}$, we determined $T$ at each point in the load curve. Then, we computed the thermal conductance, $G(T)=g_{o} T^{\beta}$ by fitting the bias power and temperature to the form $P=\left[g_{o} /(\beta+1)\right]$ $\left(T^{\beta+1}+T_{o}^{\beta+1}\right)$. Measurement of test devices with extremely small $G \sim 1 \mathrm{pW} / \mathrm{K}$ at $100 \mathrm{mK}$ sets an upper limit on the stray power of $1 \mathrm{fW}$. A compilation of all measured $G$ and corresponding $R_{\text {leads }}$ agrees with the Wiedemann-Franz law, $G \propto 1 / R_{\text {leads }}$, as expected 
since the thermal link is metallic, see Fig. 9. The Wiedemann-Franz law also predicts that metallic thermal links have $G \propto T^{1}$, or $\beta=1$, at sub Kelvin temperatures. However, the best fit values are $\beta=$ $1.6 \pm 0.2$ for bolometers with $G \leq 100 \mathrm{pW} / \mathrm{K}$. These values of $\beta$ were checked by measuring the temperature gradient due to a fixed optical load as a function of temperature. This suggests that the physics of thermal conductance of the normal metal film is modified when on the suspended beam. We find a best fit $\beta=2.7 \pm 0.36$ for bolometers with $G>$ $200 \mathrm{pW} / \mathrm{K}$. The larger $\beta \sim 2.7$ is likely influenced by nonthermal nonohmic effects [27-29]. Finally, we compute the bolometer responsivity $\bar{S}$ and NEP as a function of bias using Eqs. (1) and ().

\section{Bolometer Noise Measurement}

Noise was measured at several amplitudes of AC current bias using custom AC coupled lock-in amplifiers for all bolometers simultaneously. Usually, noise data were collected overnight when environmental disturbances were minimal. The temperature of the stages, the bolometer heat sink, regulation heater power, and room temperatures were measured and recorded synchronously with the bolometer data. The time series data files, $>1$ GByte total per night, were transferred to a different computer, deglitched, converted to a power spectrum, and averaged. The measured white noise as a function of bias, shown for a representative bolometer in Fig. 10, is in good agreement with the noise calculated using parameters from the DC load curves and Eq. (3). Absorber-less devices with $G \sim 4 \mathrm{pW} / \mathrm{K}$ were measured to validate the ability of the testbed to measure extremely low $\mathrm{NEP}<2 \mathrm{aW} / \sqrt{\mathrm{Hz}}$, which is well below the lowest $\mathrm{NEP}=9.2 \mathrm{aW} / \sqrt{\mathrm{Hz}}$ specification for the

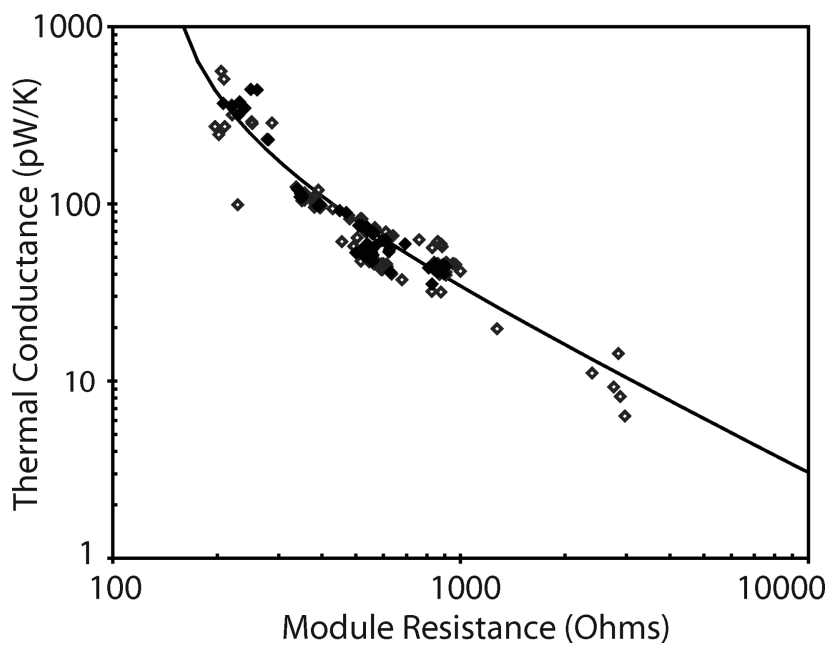

Fig. 9. Measured values of $G$ at $100 \mathrm{mK}$ as a function of modules resistance at room temperature. Solid symbols highlight the flight bolometers. The open symbols include spare and engineering model bolometers. The fitted $R_{\text {series }} \sim 10 \Omega$ is consistent with the measured resistance of the circuitry in series with the bolometer. The fitted value of $\gamma \sim 0.1$, the ratio of the bolometer lead resistance at room temperature to that at $4 \mathrm{~K}$ agrees with resistance measurements on witness samples.

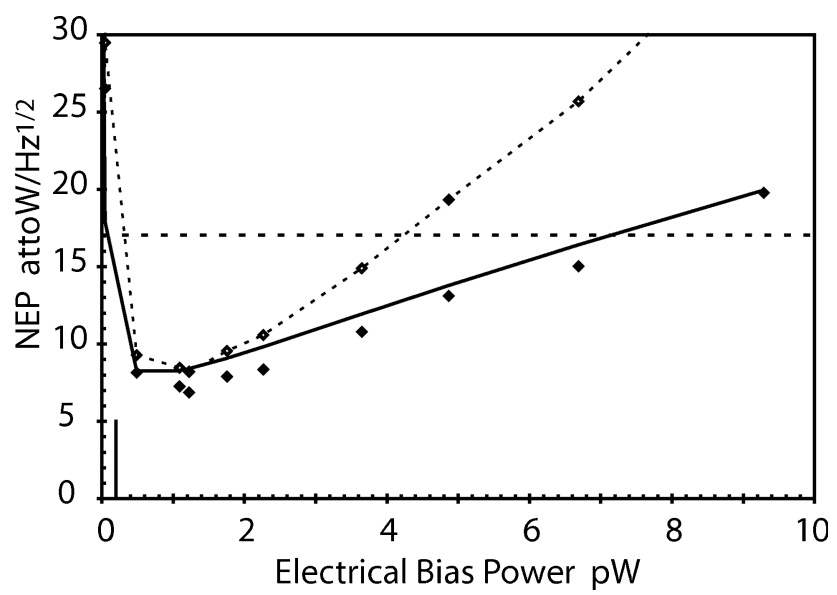

Fig. 10. Measured (solid points) and calculated (curved line) NEP as a function of bias power with the noise of the testbed amplifier subtracted. The photon noise (BLIP limit) for this band (polarized $353 \mathrm{GHz}$ ) is shown as a horizontal line. The corresponding background optical loading is shown by a vertical line on the $\mathrm{x}$ axis at $\sim 0.2 \mathrm{pW}$. "High" bias is the highest bias such that the detector and $6.5 \mathrm{nV} / \sqrt{\mathrm{Hz}}$ amplifier noise (open symbols and dashed line) is at the BLIP limit.

Planck devices. The low NEP measurements also constrain the background, nonoptical, power drift over several minutes on any bolometer to $<10 \mathrm{aW}$. The voltage of bolometers with the highest $G$, in particular the 545 and $857 \mathrm{GHz}$ devices, when at optimum bias for lowest NEP, was near the saturation voltage at the input of the lock-in amplifier. Even at a low bias, these bolometers satisfied the requirement for NEP.

\section{E. Response to Heat Sink Fluctuations}

During some noise measurements, an oscillation in the temperature of the bolometer stage was introduced to measure the coupling between the bolometer die and the heat sink. An oscillating current $\delta I_{T} e^{i \omega_{T} t}$, where $\omega_{T}=2 \pi f_{T}$ at low frequency $5<2 f_{T}<500 \mathrm{mHz}$ was applied to a resistance heater $R_{h}$ on the bolometer stage. This produced a transient response of the stage, $T(t)=T_{o}+\left(I_{T}^{2} R_{h} / 2 G_{s}\right)$ $\left[1-e^{\left(-t / \tau_{s}\right)}\right]$, starting at the time when the heater current was applied, and a sinusoidal variation in the bolometer stage temperature $\delta T_{s}=e^{i 2 \omega_{T} t}\left(\delta I_{T}^{2} R_{h}\right) /$ $\left[2\left(G_{s}+i 2 \omega_{T} C_{s}\right)\right]$. Here $\tau_{s}=C_{s} / G_{s} \sim 2000 \mathrm{~s}$ is the bolometer stage response time, $G_{s}=4 \times 10^{6} \mathrm{pW} / \mathrm{K}$ is the thermal conductance between the bolometer stage and regulated thermal intercept, and $C_{s}=8 \times$ $10^{9} \mathrm{pJ} / \mathrm{K}$ is the heat capacity of the $\sim 2 \mathrm{~kg}$ copper bolometer stage. The oscillating bolometer temperature, $\delta T_{b}\left(2 f_{T}\right)$, was determined from the peak at $2 f_{T}$ in the noise spectral density. Signal to noise $>1$ was achieved for $\delta T_{b}\left(2 f_{T}\right)>100 \mathrm{nK}$ at $2 f_{T}<$ $500 \mathrm{mHz}$. The measured $\delta T_{b}\left(2 f_{T}\right)$ was fit by a two pole model. The first and lowest pole was fixed at the value of $\tau_{s}$ determined from the transient response of the bolometer stage. The magnitude $\delta T_{b}(0)$ and a second time constant $\tau_{m}$ were free parameters in the fit. We find a range $4<\tau_{m}<7 \mathrm{~s}$ for the 
second time constant for 24 bolometers. When we compare bolometers paired in a PSB, the fore bolometer always has a lower $\tau_{m}$ compared to the aft bolometer. The value of $\tau_{m}$ roughly scales $\sim 1 / A$ for the different sized gold wires used for the backshort and housing bolometers in a PSB pair. The difference in $\tau_{m}$ for the fore and aft bolometer in a PSB pair suggests that the bolometer is heat sunk primarily through the wiring and not the copper module body.

\section{F. Optical Response Time}

We measured the optical response time of the bolometers, biased with a DC current, using a chopped infrared source. The far infrared signal was generated and modulated using a commercial blackbody source and chopper [30]. It was routed in a goldplated thin walled stainless steel light pipe in the liquid helium through a hermetically sealed quartz window into the instrument guard vacuum (IGV) can around the dilution refrigerator unit and bolometer can. The light pipe attaches to the cold quartz window flange assembly via a threaded joint, shown in Fig. 8. A brass plug was screwed into the light pipe during load curve and noise measurements and removed for optical response measurements. The plug and filters in the light pipe could be changed while the bolometers were at $100 \mathrm{mK}$ to adjust the far IR signal if necessary. A second section of the light pipe extends from the quartz window flange through the vacuum space, a second set of infrared filters at $\sim 100 \mathrm{mK}$, and then disperses the far infrared signal into the bolometer can. The optical load, chopped and unchopped, on the bolometers was in the range 0.01$1 \mathrm{pW}$ and surprisingly uniform within the bolometer can, $1-2 \mathrm{fW} / \mathrm{mm}^{2}$. The bolometer signal was demodulated at the chop frequency using commercial lockin amplifiers [31]. The signal amplitude was recorded as a function of frequency and DC bias current.

The measured bolometer signal $\delta V\left(f, I_{b}\right)=\delta V\left(I_{b}\right) /$ $\left(1+i 2 \pi \tau\left(I_{b}\right) f\right)$ was fit to Eq. (1) with $\delta V$ and $\tau$ as free parameters. The bolometer response, normalized to unity, is plotted as a function of $\tau f$ in Fig. 11. All response curves for different $\tau\left(I_{b}\right)$ collapse onto the same curve, which shows good agreement with the single pole fitting function. Some very fast $<0.5 \mathrm{~ms}$ and some very slow $\sim 100 \mathrm{~ms}$ engineering model bolometers were measured in the testbed to validate that bolometers with response times $2<\tau<8 \mathrm{~ms}$, as required for Planck, could be characterized and free of systematic errors. The values of $\tau\left(I_{b}\right)$, shown in Fig. 12, are the results of the fits of the response function to the single pole model. The heat capacity was computed using the relation $C(T)=\tau\left(I_{b}\right)[G(T)-$ $\left.\alpha P_{b}+2 \alpha P_{b} R /\left(R+R_{L}\right)\right]$ and fit to the function $C(T)=$ $C_{o} T^{\gamma}$. The best fit values of $\gamma$ range from 1.5 to 2.2 . The measured $C(T)$ at $100 \mathrm{mK}$, shown in Fig. 13, shows a significant heat capacity in excess of the calculated value expected for the absorber mesh, NTD $\mathrm{Ge}$ and contact island for all bolometer designs. The excess heat capacity scales with the surface area of the metallized $\mathrm{Si}-\mathrm{N}$ as $C_{A} \sim 1.9 \mathrm{pJ} / \mathrm{K} \mathrm{mm}^{2}$. The in-

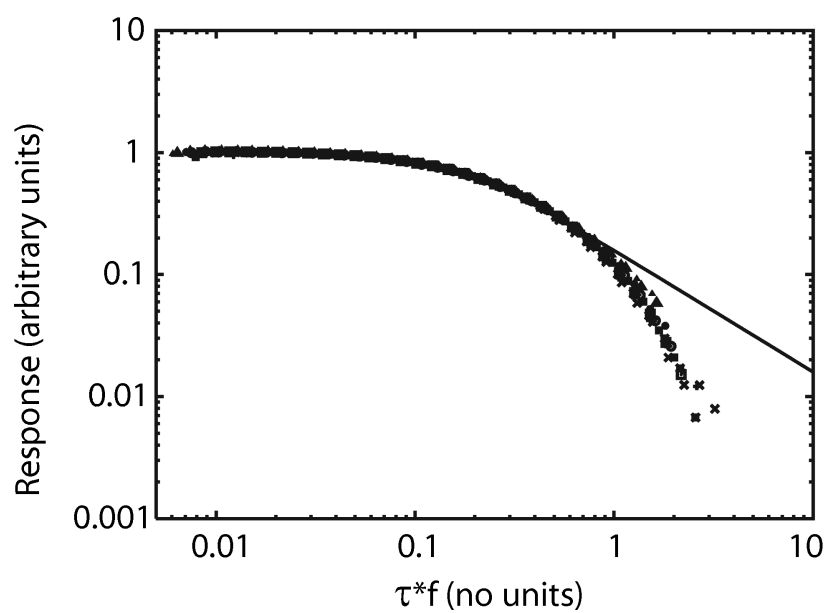

Fig. 11. Bolometer response as a function of bias for a $100 \mathrm{GHz}$ polarization sensitive bolometer. The measured response is normalized to unity, fit to a single pole roll off, and plotted as a function of $\tau f$ for a range of $4<\tau<9 \mathrm{~ms}$. The second pole from the electronic bandwidth is apparent for $\tau f>1$, which corresponds roughly to $f>150 \mathrm{~Hz}$.

tercept at $A=0$ is fixed at the measured value for the NTD Ge and contact island without any absorber at $100 \mathrm{mK}$ of $C_{\text {chip }}=0.11 \mathrm{pJ} / \mathrm{K}$. The visual appearance of the web had no correlation with excess heat capacity or relative optical efficiency in the testbed.

The excess heat capacity is not due to helium gas or other cryogenic contaminant. We determined this by always comparing a new bolometer to a reference bolometer(s) with $\tau<4 \mathrm{~ms}$ included in every $100 \mathrm{mK}$ test run. Using the reference bolometer, we were able to identify the effect of adsorbed helium in 3 of 26 test runs. In all cases when there was helium contamination, we measured $\sim 2 \mathrm{pJ} / \mathrm{K} \mathrm{mm}^{2}$ of stray heat capacity. This stray heat capacity corresponds to $\sim 0.1$ monolayer coverage of helium on the surface [32]. The presence of this adsorbed helium had no effect on the measured $G$ to the $<6 \%$ accuracy of our measurement suggesting that the sub monolayer coverage was not mobile. The source of the adsorbed

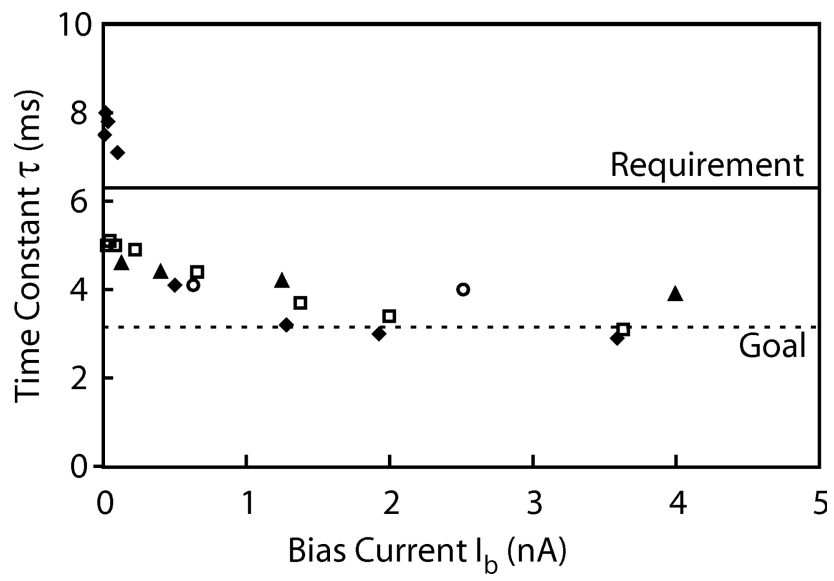

Fig. 12. Bolometer response time $\tau$ as a function of current bias at $100 \mathrm{mK}$ (solid diamonds), $139 \mathrm{mK}$ (open squares), $274 \mathrm{mK}$ (solid triangles), and $410 \mathrm{mK}$ (open circles) for a $217 \mathrm{GHz}$ SWB. 


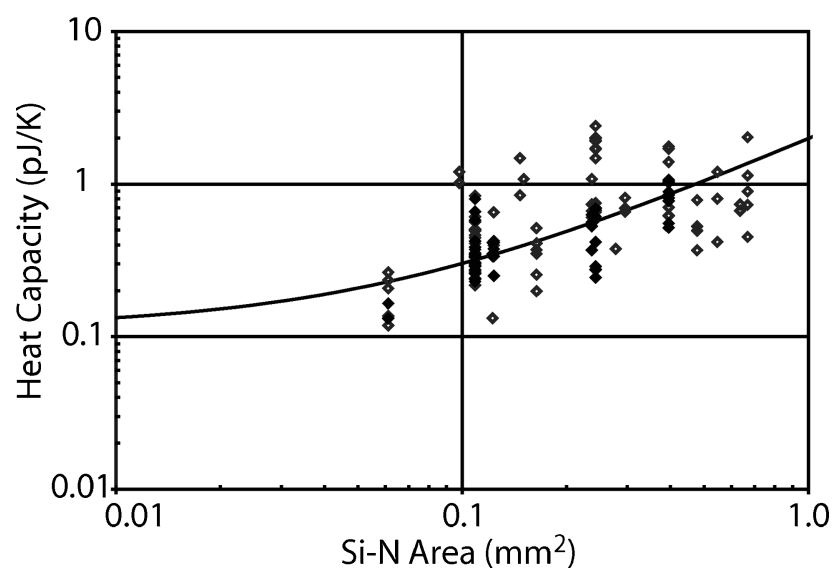

Fig. 13. Bolometer heat capacities as a function of physical absorber area and wafer. Solid symbols highlight the flight bolometers. The open symbols include spare and engineering model bolometers. The line is a linear fit to all the data which yields an excess heat capacity of $1.9 \mathrm{pJ} / \mathrm{K} \mathrm{mm}^{2}$. The intercept at $A=0$ is fixed at the heat capacity of the NTD Ge, $0.1 \mathrm{pJ} / \mathrm{K}$ measured at $100 \mathrm{mK}$ on a sample without any absorber mesh.

helium was from a leak through a poorly made indium seal of the bolometer cavity that opened and then closed while the IGV was intentionally flooded with helium gas during the cool down of the dilution insert from 300 to $4 \mathrm{~K}$. Once the system was warmed to room temperature, the indium seal was remade, and the bolometer response times remeasured, the stray heat capacity was not present. Our experience shows even submonolayer adsorption of helium can dramatically affect bolometer performance. Therefore, care must be taken to prevent helium from leaking into the vacuum space common with the bolometers.

The excess heat capacity, and therefore the time constant, proved to be the single parameter that influenced bolometer selection for flight. All other parameters, such as NEP, met flight requirements at an appropriate $I_{b}$. For bolometers designed for the $\leq 353 \mathrm{GHz}$ bands, the total heat capacity was expected to be dominated by the NTD Ge chip. Bol- ometers with the lowest excess heat capacity, as shown by filled in symbols in Fig. 13 , met the time constant requirement over a wide range of $I_{b}$ and were delivered as flight units. These bolometers were selected primarily from wafers PL217353A, 1876, 1884 , and 1921. Bolometers on other wafers, in particular 1878, were all too slow at any value of $I_{b}$ to be delivered for flight use. Bolometers for the 545 and $857 \mathrm{GHz}$ bands were selected from wafers 1905 and 1909. Bolometers for these bands differ in two ways from the lower frequency bands which enabled $100 \%$ yield to the time constant requirement. First, the bolometers are coupled to several spatial modes and these bands are more sensitive to astrophysical and instrumental foreground sources, which are warmer than the CMB. So, the background power and therefore the target $G$ for these bolometers is higher compared to the other bands, as shown in Table 3. Second, the absorber mesh filling fraction is about $3 \times$ larger than the low frequency bolometers to absorb efficiently the shorter wavelength and several spatial modes. For these more dense absorbers, the gold metallization is the dominant contribution to the heat capacity. Thus, the impact of the excess heat capacity on these wafers was inconsequential and all devices met the time constant requirement. However, without the excess, the time constants for these bolometers would have been uniformly lower $\sim 1 \mathrm{~ms}$.

The normalization constant $\delta V\left(I_{b}\right)$ was compared with the bolometer model for the responsivity $S\left(I_{b}\right)=V_{o} / \delta P$ as shown in Fig. 14. Good agreement

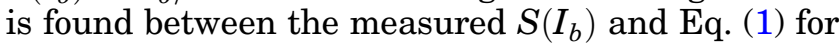
bolometers used in the bands from $100-353 \mathrm{GHz}$ which have $G<120 \mathrm{pW} / \mathrm{K}$. The measured $S\left(I_{b}\right)$ for bolometers used in the 545 and $857 \mathrm{GHz}$ bands which have $G>200 \mathrm{pW} / \mathrm{K}$ have a comparably worse fit to the calculated $S\left(I_{b}\right)$ using Eq. (1). This difference in fit quality for $G>200 \mathrm{pW} / \mathrm{K}$ is due, most likely, to nonohmic behavior of the NTD Ge [27-29].

For most bands, there is a range of bias values that satisfy both noise, responsivity, and response time requirements as shown in Fig. 10. Bolometers were selected for shipment to UCW by choosing the fastest

Table 3. Background Power and Representative DC Bias Power, NEP, and Time Constant for Bolometers Delivered to Europe ${ }^{a}$

\begin{tabular}{|c|c|c|c|c|c|c|c|}
\hline Frequency Band & $G$ & $\begin{array}{c}\text { Background } \\
\text { Power }\end{array}$ & $\begin{array}{l}\text { High Bias } \\
\text { Power }\end{array}$ & $\begin{array}{c}\text { NEP at } \\
\text { High Bias }\end{array}$ & $\begin{array}{l}\text { Bias Power } \\
\text { at Min. NEP }\end{array}$ & $\begin{array}{l}\text { Min. } \\
\text { NEP }\end{array}$ & $\begin{array}{c}\tau \text { at } \\
\text { high bias }\end{array}$ \\
\hline $\mathrm{GHz}$ & $\mathrm{pW} / \mathrm{K}$ & $\mathrm{pW}$ & $\mathrm{pW}$ & BLIP & $\mathrm{pW}$ & BLIP & $\mathrm{ms}$ \\
\hline $100(\mathrm{P})$ & 43 & 0.21 & 1.65 & 1.37 & 0.49 & 1.23 & 10.3 \\
\hline $143(\mathrm{P})$ & 53 & 0.26 & 1.8 & 1.35 & 0.48 & 1.24 & 4.2 \\
\hline 143 & 74 & 0.64 & 2.4 & 1.28 & 0.33 & 1.24 & 5.2 \\
\hline $217(\mathrm{P})$ & 60 & 0.28 & 4.2 & 1.6 & 1.1 & 1.2 & 4.3 \\
\hline 217 & 110 & 0.56 & 2.7 & 1.20 & 1.32 & 1.13 & 2.5 \\
\hline 217 & 45 & 0.56 & 2.0 & 1.18 & 0.93 & 1.11 & 3.4 \\
\hline $353(\mathrm{P})$ & 100 & 0.34 & 4.7 & 1.35 & 1.6 & 1.22 & 4.1 \\
\hline $353(\mathrm{P})$ & 60 & 0.34 & 4.0 & 1.36 & 1.1 & 1.13 & 4.2 \\
\hline 353 & 115 & 0.68 & 2.7 & 1.16 & 0.49 & 1.13 & 3.2 \\
\hline 353 & 45 & 0.68 & 2.0 & 1.12 & 0.35 & 1.09 & 1.7 \\
\hline 545 & 300 & 2.8 & 17 & 1.09 & 7 & 1.05 & 2.0 \\
\hline 857 & 300 & 10.3 & 20 & 1.02 & 7 & 1.01 & 2.4 \\
\hline
\end{tabular}

${ }^{a}$ Bands with polarization sensitivity are denoted with a (P). 


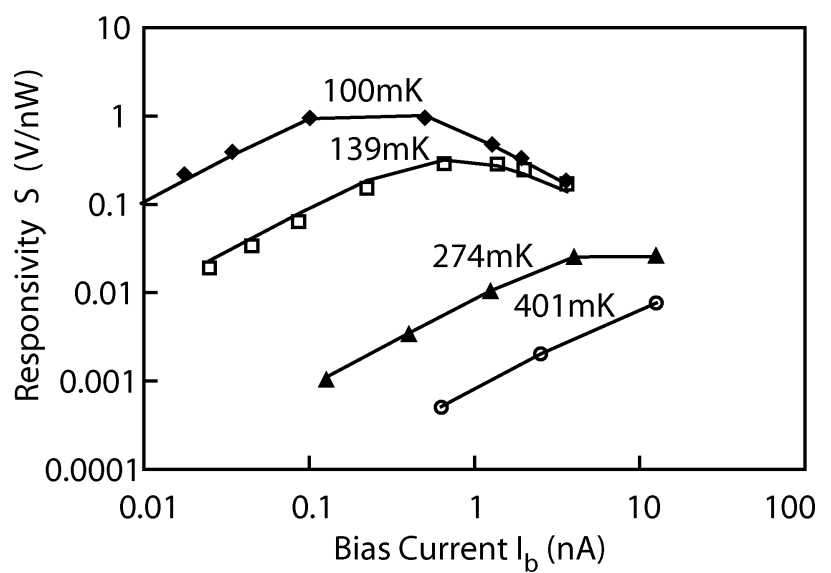

Fig. 14. Bolometer responsivity $S$ calculated from the IV curves as a function of bias and temperature (solid lines). The voltage response normalized by the chopped power was measured as a function of bias at several temperatures $100 \mathrm{mK}$ (solid diamonds), $139 \mathrm{mK}$ (open squares), $274 \mathrm{mK}$ (solid triangles), and $401 \mathrm{mK}$ (open circles) for a $217 \mathrm{GHz}$ SWB. A single value for the chopped power was determined by fitting all measured voltage responses to the calculated responsivities.

bolometers at the highest bias such that NEP and responsivity requirements were met as described in Table 3. For delivery to UCW, no official distinction between modules to be mounted in the flight focal plane and spare modules was made. The final selection was completed after an individual pixel testing at UCW. Nominal bias selection was made using a model of the complete AC-biased capacitivelycoupled bias circuit and verified during system level testing at IAS, Orsay, France.

\section{Conclusion}

The testing of the bolometers for the Planck high frequency instrument is complete. We have delivered total power and polarization sensitive bolometers designed and qualified to survive the environmental conditions of an Ariane V launch at room temperature. When cooled to $100 \mathrm{mK}$ and with the flight readout electronics, they will measure the sky in six bands from 100 to $857 \mathrm{GHz}$ at the sensitivity limit imposed by the quantum photon noise of the background radiation. Combined with the Planck low frequency instrument, the HFI will provide an increase in the sensitivity, frequency coverage, and angular resolution with respect to the in-orbit satellite WMAP. The launch of the Planck mission is planned in the first half of 2009 .

This work was performed for the Jet Propulsion Laboratory, California Institute of Technology, sponsored by the National Aeronautics and Space Administration (NASA). We acknowledge all staff at JPL who have contributed to this effort, particularly Jerry Mulder, Debi Romero, Minhee Yun, and Anarosa Villanueva for their key contributions. We also thank our collaborators at UCW, LERMA, College de France and IAS, Orsay, Paris.

\section{References}

1. J.-M. Lamarre, J. L. Puget, M. Piat, P. A. R. Ade, A. Lange, A. Benoit, P. de Bernardis, F. R. Bouchet, J. Bock, F. X. Desert, R. Emry, M. Giard, B. Maffei, A. Murphy, J. P. Torre, R. Bhatia, R. Sudiwala, and V. Yourchenko, "The Planck high frequency instrument, a 3rd generation CMB experiment and a full sky submillimeter survey," Proc. SPIE 4850, 730-739 (2002).

2. P. Mauskopf, J. Bock, H. Del Castillo, W. Holtzapfel, and A. Lange, "Composite infrared bolometers with $\mathrm{Si}_{3} \mathrm{~N}_{4}$ micromesh absorbers," Appl. Opt. 36, 765-771 (1997).

3. T. Koch, C. Paine, L. Hustead, M. Yun, A. Lange, J. Bock, R. Bhatia, W. Jones, P. Ade, and R. Sudiwala, "Bolometric detectors for the High Frequency Instrument on Planck," in Proc. IEEE Aerospace Conference (IEEE, 2002), pp. 3541-3548.

4. W. Jones, R. Bhatia, J. Bock, and A. Lange, "A polarization sensitive bolometric receiver for observations of the cosmic microwave background," Proc. SPIE 4855, 227-238 (2003).

5. W. Jones, T. Montroy, B. Crill, C. Contaldi, T. Kisner, A. Lange, C. MacTavish, C. Netterfield, and J. Ruhl, "Instrumental and analytic methods for bolometric polarimetry," Astron. Astrophys. 470, 771-785 (2007).

6. W. Holmes, J. Bock, K. Ganga, V. Hristov, L. Hustead, A. Lange, C. Paine, and M. Yun, "Preliminary performance measurements of bolometers for the Planck high frequency instrument," Proc. SPIE 4855, 208-216 (2003).

7. P. Richards, "Bolometers for infrared and millimeter waves," J. Appl. Phys. 76, 1-24 (1994).

8. J. C. Mather, "Bolometers: Ultimate sensitivity, optimization, and amplifier coupling," Appl. Opt. 23, 584-588 (1984).

9. J. C. Mather, "Bolometer noise: nonequillibrium theory," Appl. Opt. 21, 1125-1129 (1982).

10. R. V. Sudiwala, M. Griffin, and A. Woodcraft, "Thermal modelling and characterization of semiconducting bolometers," Int. J. Infrared Millim. Waves 23, 545-573 (2002).

11. E. E. Haller, "Physics and design of advanced IR bolometers and photometers," Infrared Phys. 25, 257-266 (1985).

12. J.-M. Lamarre, "Photon noise limits in mm-wave receviers," Appl. Opt. 25, 870-876 (1986).

13. S. M. Grannan, M. K. Hase, and P. Richards, "Numerical optimization of infrared bolometers," Int. J. Infrared Millim. Waves 18, 319-340 (1997).

14. D. McCammon, M. Galeazzi, D. Liu, W. Sanders, B. Smith, P. Tan, K. Boyce, R. Brekosky, J. Gygax, R. Kelley, D. Mott, F. Porter, C. Stahle, C. Stahle, and A. Szymkowiak, " $1 / f$ noise and hot electron effects in variable range hopping conduction," Phys. Status Solidi B 230, 197-204 (2002).

15. M. Yun, T. Koch, J. Bock, W. Holmes, L. Wild, J. Mulder, A. Turner, C. Paine, and A. Lange, "Bolometric detectors for the Planck surveyor," Proc. SPIE 4855, 136-147 (2003).

16. Analog Devices Belfast LTD., Hannahstown Hill, Belfast, Northern Ireland, UK, "Buried Silicon Oxide Wafers" (1999).

17. B. P. Crill, F. Piacentini, P. A. R. Ade, D. R. Artusa, R. Bathia, J. J. Bock, A. Boscaleri, P. Cardoni, S. E. Church, K. Coble, P. de Bernardis, G. deTroia, P. Farese, K. M. Ganga, M. Giacometti, E. F. Hivon, V. V. Hristov, A. Iacoangeli, W. C. Jones, A. E. Lange, L. Martinis, S. Masi, P. V. Mason, P. D. Mauskopf, L. Miglio, T. Montroy, C. B. Netterfield, C. G. Paine, E. Pascale, F. Piacentini, G. Polenta, F. Pongetti, G. Romeo, J. E. Ruhl, F. Scaramuzzi, D. Sforna, and A. D. Turner, "Boomerang: A balloon-borne millimeter-wave telescope and total power receiver for mapping anisotropy in the cosmic microwave background," Astrophys. J. Suppl. Ser. 148, 527-541 (2003).

18. C. Paine, J. Bock, V. Hristov, and A. Lange, "A low noise, high thermal stability, $0.1 \mathrm{~K}$ test facility for the Planck HFI bolometers," in Advances in Cryogenic Engineering, L. Summers, ed. (Plenum, 2001), Vol. 47, pp. 1651-1658. 
19. F. Piacentini, P. A. R. Ade, R. Bathia, J. J. Bock, A. Boscaleri, P. Cardoni, B. P. Crill, P. de Bernardis, H. Del Castillo, G. De Troia, P. Farese, M. Giacometti, E. F. Hivon, V. V. Hristov, A. Iacoangeli, A. E. Lange, S. Masi, P. D. Mauskopf, L. Miglio, C. B. Netterfield, P. Palangio, E. Pascale, A. Raccanelli, S. Rao, G. Romeo, J. Ruhl, and F. Scaramuzzi, "The Boomerang North America Instrument: A balloon-borne radiometer optimized for measurements of the cosmic microwave background radiation from 0.3 degrees to 4 degrees," Astrophys. J. Suppl. Ser. 138, 315-336 (2002).

20. Stanford Research Systems, Inc., "SRS360 Low Distortion Function Generator," http://www.thinksrs.com/products/ DS360.htm (2000).

21. National Instruments Corporation, 11500 N Mopac Expwy Austin, TX, "NI PCI-6031E Multifunction Digital Acquisition Board," (2002).

22. Lake Shore Cryotronics, Inc., "Germanium Resistance Thermometers," http://www.lakeshore.com/temp/sen/gtrd .html (2000).

23. PICOWATT (RV-ELEKTRONIIKKA OY), "AVS-47 AC Resistance Bridge," http://www.picowatt.fi/index1.html (2000).

24. Haller Beeman Assoc., "NTD Thermometer Type I," http:// www.haller-beeman.com/ (2000).
25. PICOWATT (RV-ELEKTRONIIKKA OY), "TS-530A Temperature Controller," http://www.picowatt.fi/index1.html (2000).

26. M. Griffin and W. Holland, "Bolometer optimization," Int. J. Infrared Millim. Waves 9, 861-875 (1988).

27. M. Piat, J.-P. Torre, E. Breelle, A. Coulais, A. Woodcraft, W. Holmes, and R. Sudiwala, "Modeling of Planck high frequency instrument bolometers using non-linear effects in the thermometers," Nucl. Instrum. Methods Phys. Res. A 559, 588-590 (2006).

28. T. Kenny, P. Richards, I. Park, E. Haller, and J. Beeman, "Biasinduced non-linearities in the dc I-V characteristics of neutron transmutation-doped germanium at liquid- ${ }^{4} \mathrm{He}$ temperatures," Phys. Rev. B 39, 8476-8482 (1989).

29. S. Grannan, A. Lange, E. Haller, and J. Beeman, "Non-ohmic hopping conduction in doped germanium at $T<1 \mathrm{~K}$," Phys. Rev. B 45, 4516-4519 (1992).

30. Infrared Systems Development Corp., "IR-508/301 Blackbody System," http://www.infraredsystems.com/ (2000).

31. Stanford Research Systems, Inc., "SRS530 Analog Lock-In Amplifier," http://www.thinksrs.com/products/SR510530.htm (2000).

32. J. Birmingham and P. Richards, "The heat capacity of ${ }^{4} \mathrm{He}$ monolayers adsorbed on evaporated gold," J. Low Temp. Phys. 109, 267-286 (1997). 